\title{
Microflora fúngica de sementes comerciais de Panicum maximum e Stylosanthes spp.
}

\author{
Fungic microflora of Panicum maximum and \\ Styosanthes spp. commercial seed
}

\author{
Carlos Eduardo Marchi ${ }^{*}$; Celso Dornelas Fernandes ${ }^{2}$; Marcelo Leandro Bueno ${ }^{3}$; \\ Margareth Vieira Batista ${ }^{2}$; Larissa Rodrigues Fabris ${ }^{4}$
}

\begin{abstract}
Resumo
Sementes comerciais de forrageiras tropicais, pertencente a 26 lotes produzidos em diferentes regiões (safras 2004-05 e 2005-06), foram avaliadas quanto à sanidade. Foram analisadas sementes de cultivares de Panicum maximum (Massai, Mombaça e Tanzânia) e de estilosantes (Estilosantes Campo Grande - ECG). Adicionalmente, avaliou-se a qualidade sanitária de dois lotes de sementes de P. maximum destinados à exportação. Para isso, as sementes foram submetidas ao teste de papel de filtro em gerbox, os quais foram incubados a $20^{\circ} \mathrm{C}$, com fotoperíodo de $12 \mathrm{~h}$, durante sete dias. Os fungos saprófitos ou secundários (FSS) mais frequentemente detectados nos lotes de P. maximum foram Aspergillus sp., Cladosporium sp. e Rhizopus sp., os quais, em geral, apresentaram baixa incidência nas sementes. Quanto aos fungos patogênicos (FP), constatou-se elevado número de lotes contaminados pelos gêneros Bipolaris, Curvularia, Fusarium e Phoma. Em geral, foi elevada a incidência de FP nas sementes de P. maximum. A ocorrência de Phoma sp. foi a mais crítica, pois $81 \%$ dos lotes registraram índices superiores a 50\%. Nas sementes de 'ECG' foram detectados FSS (gêneros Aspergillus, Cladosporium e Penicillium) e FP (gêneros Bipolaris, Curvularia, Fusarium e Phoma), os quais apresentaram, em geral, baixa incidência. FSS e FP também foram associados às sementes de $P$. maximum tipo exportação, em alguns casos, com incidência considerável. Os resultados indicaram que em todas as regiões produtoras existiu pelo menos um fator limitante afetando a qualidade sanitária das sementes.

Palavras-chave: Capim guiné, estilosantes, patologia de sementes, fungos, qualidade sanitária
\end{abstract}

\begin{abstract}
The sanitary quality of 26 lots commercial seeds of tropical forages, produced in different regions (2004-05 and 2005-06) was analyzed. The lots were composed of seeds of Panicum maximum ('Massai', 'Mombaça' e 'Tanzânia') and stylo ('Estilosantes Campo Grande' - ECG). Additionally, seeds of two lots of $P$. maximum for exportation were analyzed. The blotter test was used, at $20^{\circ} \mathrm{C}$ under alternating light and darkness in a $12 \mathrm{~h}$ photoperiod, for seven days. The Aspergillus, Cladosporium and Rhizopus genus consisted the secondary or saprophytes fungi (FSS) with greatest frequency in P. maximum lots. In general, there was low incidence of these fungus in the seeds. In relation to pathogenic fungi (FP),
\end{abstract}

${ }^{1}$ Laboratório Nacional Agropecuário em São Paulo (Lanagro-SP), Ministério da Agricultura, Pecuária e Abastecimento (MAPA), CEP 13208-051, Jundiaí, SP, Brasil. E-mail: carlos.marchi@agricultura.gov.br

2 Embrapa Gado de Corte, Cx. Postal 154, CEP 79002-970, Campo Grande, MS, Brasil. E-mail: celsof@cnpgc.embrapa.br; margareth@cnpgc.embrapa.br

${ }^{3}$ Mestrando em Biologia Vegetal, Universidade Federal do Mato Grosso do Sul, Cx. Postal 549, CEP 79070-900, Campo Grande, MS, Brasil. E-mail: buenobio@yahoo.com.br

${ }^{4}$ Mestranda em Saúde e Desenvolvimento na Região Centro-Oeste, Universidade Federal do Mato Grosso do Sul, Cx. Postal 549, CEP 79070-900, Campo Grande, MS, Brasil. E-mail: laribio@hotmail.com

* Autor para correspondência 
it was detected high frequency of contaminated lots by Bipolaris, Curvularia, Fusarium and Phoma genus. Generally, there was high incidence of FP in P. maximum seeds. The occurrence of Phoma sp. was hight, because in $81 \%$ of the lots showed incidence superior to $50 \%$. In 'ECG' seeds it was detected FSS (Aspergillus, Cladosporium, and Penicillium genus) and FP (Bipolaris, Curvularia, Fusarium and Phoma genus), usually, in low incidence. FSS and FP were associated to P. maximum seeds for exportation, with significant incidence in some cases. The results indicated that there was a limiting factor in all producer regions regarding sanitary quality of the seeds.

Key words: Guinea grass, stylo, pathology of seeds, fungi, sanitary quality

\section{Introdução}

A sanidade da semente constitui fator importante para o estabelecimento e a manutenção de pastagens tropicais com boa qualidade. Entretanto, é comum o emprego de sementes com baixa qualidade sanitária, sobretudo no mercado interno. Dentre os fatores favorecedores, destacam-se a ausência de padrões sanitários na comercialização interna de sementes e a indiferença do pecuarista que toma como base o preço por quilograma como critério para aquisição de sementes, o que não estimula a adequação do setor (MARCHI et al, 2006b).

Uma vez presentes nas sementes, os patógenos podem afetar a germinação, o desenvolvimento das plântulas, a produção de sementes e a longevidade das forrageiras no campo, além de prejudicar as exportações das mesmas (FERNANDES et al, 2005; VECHIATO; APARECIDO, 2008). Como as sementes contaminadas constituem mecanismo eficiente de introdução e dispersão de patógenos, a maior parte dos países importadores de sementes de forrageiras impõe restrições fitossanitárias ao insumo brasileiro (TSUHAKO, 2009).

Recentemente, a análise sanitária de sementes comerciais de braquiária, destinadas ao mercado interno, revelou a presença de importantes agentes fitopatogênicos, como Bipolaris sp., Curvularia sp., Fusarium sp. e Phoma sp. (GUIMARÃES et al., 2006). Também a presença de Ustilago operta, agente causal do carvão, foi reportada em sementes comerciais de Brachiaria brizantha
('Marandu' e 'Xaraés') e Brachiaria decumbens cv. Basilisk (MARCHI et al., 2009). Em adição, foram constatadas incidências consideráveis dos gêneros Bipolaris, Fusarium e Phoma em sementes de 'Maraundu', 'Xaraés' e 'Basilisk' com alto padrão de pureza, destinadas ao mercado externo (GUIMARÃES et al., 2006).

Para o Panicum maximum e Stylosanthes spp. são escassas informações sobre fungos veiculados às sementes comerciais, o que pode contribuir para o aumento da incidência de doenças nas pastagens. No sentido de ampliar esse conhecimento, procedeuse a análise sanitária de sementes das principais cultivares de $P$. maximum e de Stylosanthes capitata e Stylosanthes macrocephala, componentes da cv. Estilosantes Campo Grande, produzidas em diferentes regiões do país e comercializadas no mercado interno. Também se avaliou a incidência de fungos em lotes de sementes de $P$. maximum com alta pureza física, destinados ao mercado externo.

\section{Material e Métodos}

\section{Amostras de sementes}

As sementes de lotes (L) comerciais de $P$. maximum e de $S$. capitata e $S$. macrocephala, componentes da cv. Estilosantes Campo Grande (ECG), destinadas ao mercado interno, foram fornecidas por empresas do setor. Estas foram produzidas nas safras 2004-05 e 2005-06, em diferentes regiões produtoras do país (Tabela 1). 
Tabela 1. Sementes de lotes comerciais de Panicum maximum ('Massai', 'Mombaça' e 'Tanzânia') e de 'Estilosantes Campo Grande' (mistura física de Stylosanthes capitata e Stylosanthes macrocephala), com as respectivas origens e ano de produção, submetidas à análise sanitária pelo método do papel de filtro.

\begin{tabular}{cccc}
\hline \multirow{2}{*}{ Cultivar } & Lote & \multicolumn{2}{c}{ Origem / Safra } \\
\cline { 3 - 4 } & (L) & $\mathbf{2 0 0 4 - 0 5}$ & 2005-06 \\
\cline { 3 - 4 } P. maximum 'Mombaça' & $\mathbf{1}$ & Jales-SP & Jales - SP \\
& $\mathbf{2}$ & NI & Paraíso - MS \\
& $\mathbf{4}$ & Paraíso-MS & Camapuã - MS \\
& $\mathbf{1}$ & NI (SP) & Rondonópolis - MT \\
\hline P. maximum 'Tanzânia' & $\mathbf{2}$ & Auriflama-SP & Paranapuã - SP \\
& $\mathbf{3}$ & NI (SP) & Rondonópolis - MT \\
& $\mathbf{4}$ & Paraíso - MS & Uberlândia - MG \\
& $\mathbf{5}$ & Guzolândia - SP & Guzolândia - SP \\
\hline \multirow{2}{*}{ maximum 'Massai' } & $\mathbf{1}$ & Água Clara - MS & - \\
\hline S. capitata e $S$. & $\mathbf{2}$ & Paraíso-MS & Rondonópolis - MT \\
macrocephala & $\mathbf{1}$ & NI & Rondonópolis - MT \\
('Estilosantes Campo & $\mathbf{2}$ & Paraíso-MS & Camapuã - MS \\
Grande') & $\mathbf{3}$ & NI & Paraíso - MS \\
\hline
\end{tabular}

*Local de produção não informado.

Sementes de dois lotes comercias de P. maximum com alta pureza física, produzidas na safra 2004-05, foram analisadas quanto à qualidade sanitária. Um lote, pertencente à cv. Tanzânia, foi produzido em Auriflama-SP, enquanto o outro, pertencente à cv. Mombaça, foi composto por sementes produzidas em São Francisco Sales-MG, Paranapuã-SP e Quirinópolis-GO.

Detecção e identificação dos fungos associados às sementes

Para a avaliação da microflora fúngica associada às sementes utilizou-se o método do papel de filtro (Blotter Test). Foram analisadas 200 sementes de cada cultivar, estas divididas em 10 gerbox (20 sementes/ gerbox). As sementes, após dispostas sobre duas folhas de papel de filtro estéril umedecido, com água destilada e esterilizada, foram incubadas durante sete dias sob a temperatura de $20^{\circ} \mathrm{C}$ e alternância de 12 horas de luz. Em seguida, procedeu-se a identificação dos fungos desenvolvidos nas sementes sob microscópio estereoscópico e de luz. Quando necessário, recorreu-se à literatura pertinente para auxílio na identificação dos fungos.

\section{Análise dos dados}

Os procedimentos estatísticos foram realizados com o programa SAS (SAS INSTITUTE, 2003). Para atender as pressuposições da análise de variância os dados de incidência (\%) de fungos nas sementes foram transformados para $\sqrt{(x+0,01)}$. Para a interpretação dos resultados, foram apresentadas as médias dos dados originais. As comparações múltiplas entre as médias foram realizadas pelos testes de Tukey a 5\% de probabilidade.

\section{Definições operacionais}

O termo "fungo patogênico" foi empregado para caracterizar os fungos capazes de incitar doença em forrageiras tropicais, ou que, embora não demonstrado, apresentam potencial para tanto. A designação "fungos saprofíticos ou secundários" foi designada para os fungos que apresentam menor 
importância econômica para as forrageiras tropicais ou que podem ser responsáveis pela deterioração de sementes durante o armazenamento (fungos de armazenamento).

\section{Resultados e Discussão}

Fungos associados às sementes comerciais de $P$. maximum

Os fungos saprófitos ou secundários (FSS) mais detectados nos lotes de P. maximum foram Aspergillus sp, Rhizopus sp. e Cladosporium sp. (Tabela 2). Também foram observados lotes contaminados pelos gêneros Alternaria, Nigrospora e Penicillium, porém com menor frequência. Epicoccum sp. foi detectado apenas nos lotes de sementes produzidos em 2004-05, em 54,5\% destes. Esses gêneros de fungos frequentemente têm sido descritos associados às sementes de $P$. maximum (MARCHI et al., 2006a; GOMES et al., 2008), sendo que os gêneros Aspergillus, Cladosporium e Rhizpous já foram detectados anteriormente em sementes importadas do Japão (URBEN, 2004).

Tabela 2. Porcentagem de lotes comerciais de sementes de Panicum maximum ('Massai', 'Mombaça' e 'Tanzânia') e de 'Estilosantes Campo Grande' (mistura física de Stylosanthes capitata e Stylosanthes macrocephala), produzidos em diferentes regiões do Brasil nas safras 2004-05 e 2005-06, contaminados por fungos.

\begin{tabular}{|c|c|c|c|c|c|c|c|c|}
\hline \multirow{3}{*}{ Fungo } & \multicolumn{6}{|c|}{ P. maximum } & \multirow{2}{*}{\multicolumn{2}{|c|}{$\begin{array}{c}\text { 'Estilosantes Campo } \\
\text { Grande' }\end{array}$}} \\
\hline & \multicolumn{2}{|c|}{ 'Massai' } & \multicolumn{2}{|c|}{ 'Mombaça' } & \multicolumn{2}{|c|}{ 'Tanzânia' } & & \\
\hline & $2004-05^{1}$ & $2005-06^{1}$ & $2004-05^{2}$ & $2005-06^{2}$ & $2004-05^{2}$ & $2005-06^{3}$ & 2004-05' & $2005-06^{4}$ \\
\hline Alternaria alternata & - & - & 25 & 50 & 60 & - & 0,0 & 0,0 \\
\hline Aspergillus sp. & $50^{*}$ & 100 & 100 & 100 & 80 & 50 & 0,0 & 100,0 \\
\hline Bipolaris sp. & 50 & 100 & 100 & 100 & 100 & 100 & 0,0 & 100,0 \\
\hline Cladosporium sp. & - & 100 & 25 & 75 & 80 & - & 0,0 & 33,3 \\
\hline Curvularia sp. & 50 & 100 & 100 & 100 & 100 & 100 & 0,0 & 33,3 \\
\hline Epicoccum sp. & - & - & 50 & - & 80 & - & 0,0 & 0,00 \\
\hline Fusarium sp. & 100 & 100 & 100 & 100 & 100 & 100 & 100,0 & 100,0 \\
\hline Nigrospora sp. & - & - & 25 & 25 & 20 & 25 & 0,0 & 0,00 \\
\hline Penicillium sp. & - & 50 & 50 & - & - & - & 0,0 & 33,3 \\
\hline Phoma sp. & 100 & 100 & 100 & 100 & 100 & 100 & 100,0 & 100,0 \\
\hline Rhizopus sp. & 50 & 100 & 75 & 100 & 20 & 25 & 0,0 & 0,0 \\
\hline
\end{tabular}

- Não detectado na cultivar. *Porcentagem de lotes contaminados. Determinado pelo método do papel de filtro. 1 Dois lotes de sementes analisados. $\stackrel{2}{=}$ Quatro lotes de sementes analisados. $\stackrel{3}{ }$ Cinco lotes de sementes analisados. ${ }^{4}$ Três lotes de sementes analisados. 
$\mathrm{Na}$ maior parte dos lotes de sementes de $P$. maximum se constatou baixa incidência de FSS, em ambas as safras (Tabelas 3 e 4). Na safra 2004-05, o maior índice foi cerca de 4,0\%, correspondente à associação de Aspergillus sp. e 'Tanzânia' (L4) (Tabela 3). Com algumas exceções, a baixa incidência ou ausência de FSS, levou a não diferenciação dos lotes de sementes avaliados. $\mathrm{Na}$ safra 2005-06, somente dois lotes de sementes, pertencentes à 'Mombaça' (L1 e L2), apresentaram percentuais de incidência de FSS maiores que 10,0\%, especificamente para Aspergillus sp. (Tabela 4). Essa maior incidência pode ser devido às práticas na colheita e pós-colheita, bem como à umidade relativa do ar e demais cuidados durante o período de armazenamento (FARIAS et al., 2002). A contaminação das sementes por Aspergillus sp. e Rhizopus sp. é preocupante, uma vez que esses podem causar deterioração de sementes, e reduzir a germinação e o vigor das mesmas (CIRIO; LIMA, 2003; BARRETO et al., 2004).

Para os fungos patogênicos (FP), os mais frequentemente detectados nos lotes de sementes avaliados foram dos gêneros Bipolaris, Curvularia, Phoma e Fusarium (Tabela 2). Eles foram detectados em $100 \%$ dos lotes de sementes da safra 200506. Os gêneros supracitados têm sido comumente encontrados associados às sementes de $P$. maximum (GARCIA; TENENTE, 2000; MARCHI et al., 2006a; MICHALSKI et al., 2007). Os FP são observados nas áreas de produção de sementes e presentes em grande parte do ciclo da cultura.

Em geral, constatou-se elevada incidência de FP nas sementes de P. maximum (Tabelas 3 e 4). Nas sementes de 'Tanzânia' (L1, da safra 200405), a incidência de Bipolaris sp. e Curvularia sp. foi de $64,5 \%$ e $48,5 \%$, respectivamente (Tabela 3). Também foram observados percentuais elevados de Fusarium sp., principalmente nas sementes de L2 de 'Massai' (safra 2004-05). Para Phoma sp., foram observados percentuais de incidência superiores a 50\% em 17 dos 21 lotes de sementes analisados (Tabelas 3 e 4). No caso de L2 de 'Massai' (da safra 2005-06), o percentual de sementes com Phoma sp. foi de $85,5 \%$ (Tabela 4). Em alguns casos, os níveis de incidência desses FP nas sementes dos diversos lotes de $P$. maximum foram semelhantes, o que não permitiu observar-se diferença entre os mesmos (Tabelas 3 e 4).

Aalta porcentagem de lotes contaminados (Tabela 2) e a elevada incidência nas sementes (Tabelas 3 e 4), de ambas as safras, indicaram que Bipolaris sp., Fusarium sp. e Phoma sp. constituem os FP mais comuns nos campos de produção de sementes de P. maximum. Eles têm sido apontados como sendo os principais FP associados às sementes comerciais de braquiária (GUIMARÃES et al., 2006). Esses mesmos autores apontaram a inexistência de padrões de qualidade sanitária para a comercialização das sementes de forrageiras tropicais, bem como a falta de produtos registrados para tratamento das sementes como sendo os fatores responsáveis pela ampla dispersão dos FP.

Os elevados percentuais de FP observados nas sementes das duas safras podem ter como causa às condições climáticas favoráveis nas regiões produtoras. Também a produção de modo continuado em mesma área e/ou em áreas com histórico de ocorrência de FP pode favorecer ao aumento da incidência desses patógenos, devido ao incremento do inóculo na área (FERNANDES et al., 2005). 


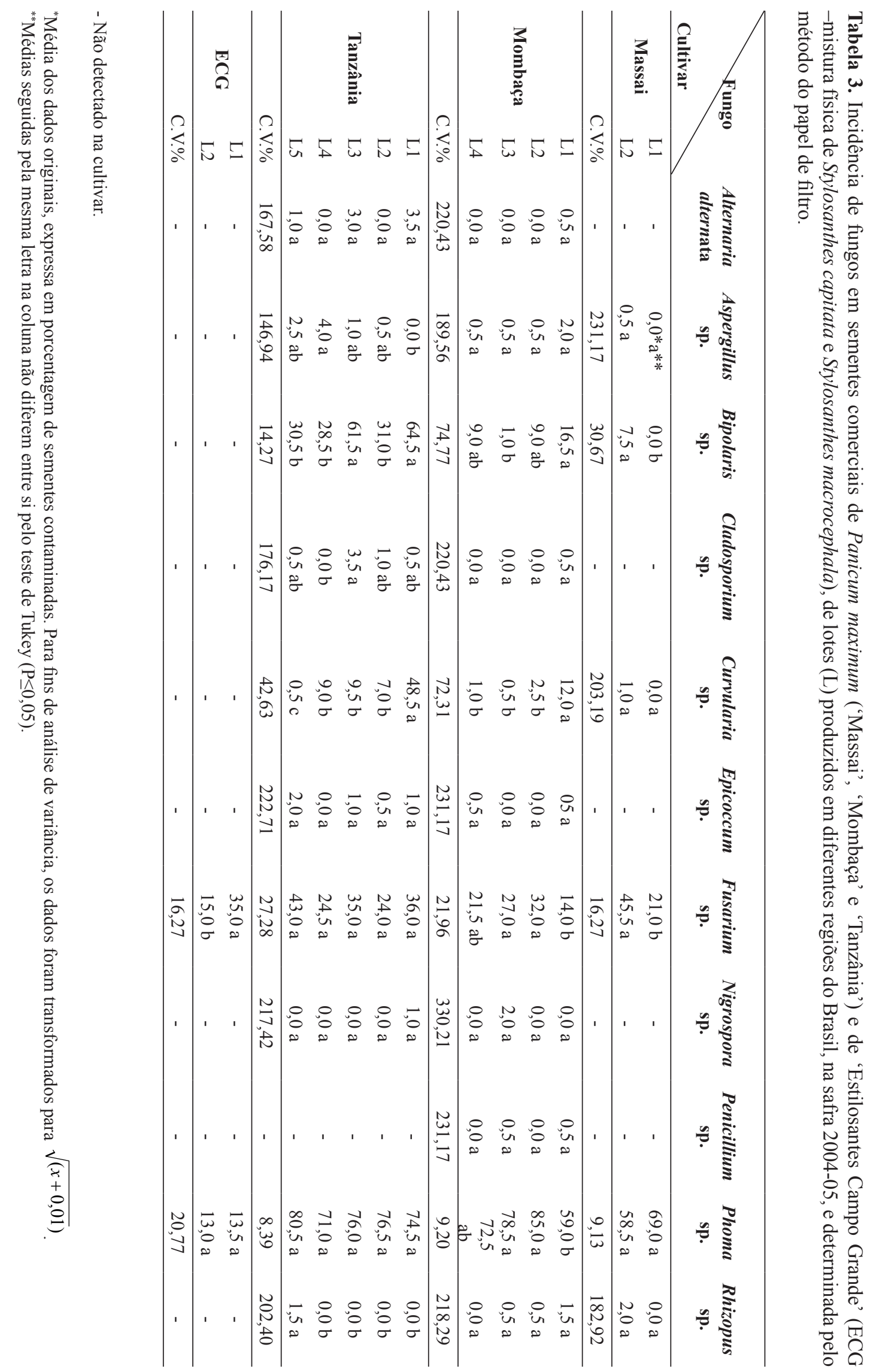




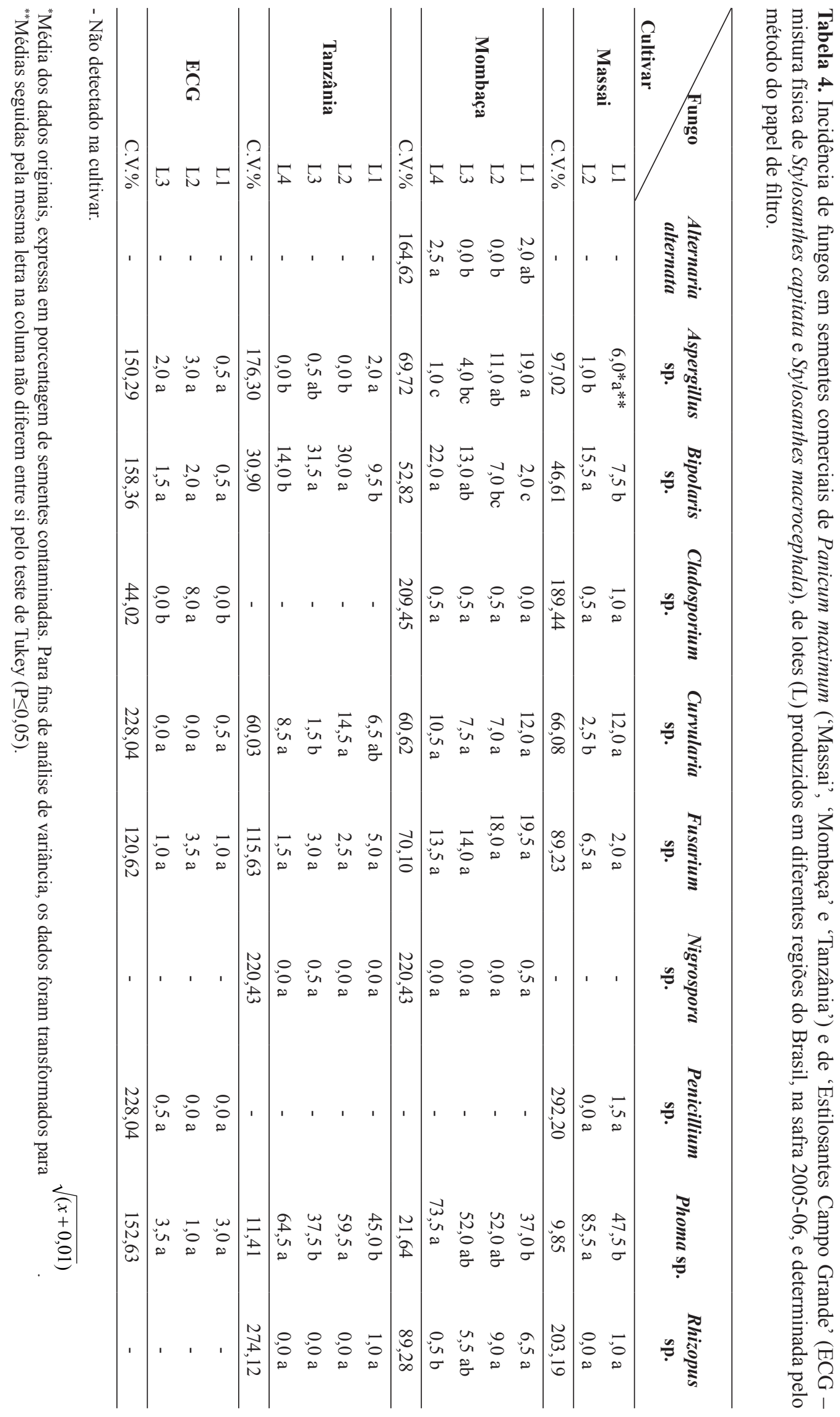


Os índices de FP detectados nas sementes de $P$. maximum são motivos de preocupação, pois, alguns desses fungos podem reduzir a viabilidade das sementes (NEERGAARD, 1979), e outros com crescimento rápido e agressivo, a exemplo de Fusarium sp. e Phoma sp., podem causar a morte das sementes mesmo antes da germinação (MENTEN, 1995). Podem ser transmitidos pela semente e causar doença em plântulas. Michalski et al. (2007) observaram a transmissão de Bipolaris sp. e Fusarium sp. das sementes de P. maximum infectadas naturalmente para a raiz e o sistema basal das plântulas até 50 dias após a semeadura. Para sementes de braquiária, Lasca, Vechiato e Kohara (2004) apresentaram evidências da transmissão de Curvularia sp. e Phoma sp. Para Bipolaris sp., a sua capacidade de transmissão e a elevada incidência em sementes comerciais, poderiam, em parte, justificar o aumento de manchas foliares causadas pelo patógeno em áreas de produção de $P$. maximum (MARCHI et al., 2006b).

Pelos percentuais de incidência total de FSS e FP nas sementes de $P$. maximum produzidas em 200405 , evidenciou-se maior qualidade sanitária em L1 de 'Massai', L4 de 'Mombaça' e L4 de 'Tanzânia' (Tabela 3). Menor sanidade foi verificada em L2 'Massai', L2 de 'Mombaça' e L1 de 'Tanzânia'. Para a safra 2005-06, L1 de 'Massai', L3 de 'Mombaça' e L1 de 'Tanzânia' apresentaram melhor qualidade sanitária (Tabela 4). O mesmo não foi observado para L2, L4 e L2 de 'Massai', 'Mombaça' e 'Tanzânia', respectivamente, cujas sementes apresentaram maiores percentuais de fungos associados.

Pelos resultados obtidos, não foi possível considerar nenhuma das regiões produtoras como destaque, provavelmente pela existência de pelo menos um fator limitante para a produção de sementes com qualidade sanitária satisfatória. Sobretudo, os elevados percentuais de Phoma sp. conferiram menor qualidade sanitária às sementes de $P$. maximum produzidas nas safras de 2004-05 e 2005-06 (Tabelas 3 e 4). Resultados semelhantes foram observados para as sementes comerciais de braquiária (GUIMARÃES et al., 2006).

Para as sementes de $P$. maximum destinadas ao mercado externo foram detectados FSS, especificamente Aspergillus sp. e Rhizopus sp. e FP dos gêneros Bipolaris, Fusarium e Phoma, em geral, com maiores percentuais de incidência nas sementes de 'Mombaça' e melhor qualidade sanitária para as sementes de 'Tanzânia' (Tabela 5). Espécies de FSS e FP também foram observadas por Guimarães al. (2006) em lotes de sementes de braquiária destinados à exportação. Mesmo sob condições como melhor qualidade física (pureza acima de 90\%), ou seja, menor conteúdo de impurezas (resíduos vegetais e solo), não têm sido observados índices aceitáveis de incidência de fungos nas sementes destinadas à exportação. A presença de patógenos em sementes tipo exportação pode-se constituir em um entrave para as exportações brasileiras, mesmo porque os países importadores impõem restrições fitossanitárias à entrada de produtos de origem vegetal. A constatação de sementes de gramíneas forrageiras veiculando Claviceps maximensis e/ ou Claviceps purpurea, por exemplo, representa barreira fitossanitária às transações comerciais com a Guatemala, México e Panamá (ALVES; DIAS, 2000; TSUHAKO, 2009). 
Tabela 5. Incidência de fungos em sementes comerciais de Panicum maximum 'Mombaça' e 'Tanzânia', de lotes com alta pureza física e destinados à exportação, produzidos na safra 2004-05, determinada pelo método do papel de filtro.

\begin{tabular}{cccccc}
\hline Fungo & Aspergillus sp. & Bipolaris sp. & Fusarium sp. & Phoma sp. & Rhizopus sp. \\
\hline $\begin{array}{c}\text { P. maximum } \\
\text { 'Mombaça'1 }\end{array}$ & $1,0^{*}$ & 14,0 & 26,0 & 9,5 & 2,0 \\
$\begin{array}{c}\text { P. maximum } \\
\text { 'Tanzânia' }\end{array}$ & 0,5 & 5,5 & 13,0 & 9,0 & 0,0 \\
\hline
\end{tabular}

"Média expressa em porcentagem de sementes contaminadas. ${ }^{1}$ Sementes produzidas em São Francisco Sales-MG, Paranapuã-SP e Quirinópolis-GO. ${ }^{2}$ Sementes produzidas em Auriflama-SP.

Fungos associados às sementes de Estilosantes Campo Grande

Os lotes de sementes da leguminosa forrageira cv. Estilosantes Campo Grande (ECG), composta pela mistura física de Stylosanthes capitata e S. macrocephala, produzidos em 2004-05, apresentaram apenas contaminações por FP (Tabela 3) Foram observadas sementes contaminadas por Fusarium sp. e Phoma sp., cujos maiores percentuais foram de $35,0 \%$ e $13,5 \%$, respectivamente, para as sementes do L1 (Tabela 3). Na safra 2005-06, foram detectados os fungos Bipolaris sp., Curvularia sp., Fusarium sp. e Phoma sp., além de FSS (Aspergillus sp., Cladosporium sp. e Penicillium sp.). Em geral, esses gêneros de fungos também foram detectados por Urben (2004) em sementes de Stylosanthes sp. oriundas da Austrália.

Os FSS foram observados em baixos percentuais nas sementes produzidas em 2005-06 (Tabela 4). O maior percentual foi de $8,0 \%$ para Cladosporium sp. nas sementes do L2. Também nesta safra, a incidência de FP foi baixa e o maior percentual $(2,0 \%)$ foi para o fungo Bipolaris sp. nas sementes do L2.

Nas sementes de 'ECG' de ambas as safras não foi observada a presença do Colletotrichum gloeosporioides, agente causal da antracnose de estilosantes, tampouco da forma teliomórfica do mesmo, Glomerella cingulata. Esses fungos foram observados por Marchi et al. (2006a) em sementes de estilosantes não escarificadas mecanicamente.
Possivelmente, o processo de escarificação, realizado nas sementes comerciais para superar a dormência física, possa contribuir na redução da incidência desses patógenos.

\section{Conclusões}

Fungos como Aspergillus sp. e Rhizopus sp. (FSS) e Bipolaris sp., Fusarium sp. e Phoma sp. (FP) são predominantes em sementes de P. maximum 'Massai', 'Mombaça' e 'Tanzânia' e de 'Estilosantes Campo Grande' ( $S$. capitata e $S$. macrocephala), mesmo em condições de elevada pureza física. Quanto às regiões produtoras, nenhuma se destaca quanto à qualidade sanitária das sementes produzidas.

\section{Agradecimentos}

À Fundação de Apoio ao Desenvolvimento do Ensino, Ciência e Tecnologia do Estado de Mato Grosso do Sul (Fundect-MS), à Fundação Manoel de Barros (FMB-MS) e ao Conselho Nacional de Desenvolvimento Científico e Tecnológico (CNPq), pelo apoio financeiro à pesquisa. À Embrapa Gado de Corte, pela oportunidade da realização do trabalho.

\section{Referências}

ALVES, S. J.; DIAS, M. C. L. Contribuições da pesquisa à produção de sementes de espécies forrageiras IAPAR. In: WORKSHOP SOBRE SEMENTES DE 
FORRAGEIRAS, 1., 1999, Sete Lagoas. Anais... Sete Lagoas: Embrapa Negócios Tecnológicos, 2000. p. 7380.

BARRETO, A. F.; EGBERTO, A.; BONIFÁCIO, B. F.; FERREIRA, O. R. R. S.; BELÉM, L. F. Qualidade fisiológica e a incidência de fungos em sementes de algodoeiro herbáceo tratadas com estratos de agave. Revista Brasileira de Oleaginosas e Fibrosas, Campina Grande, v. 8, n. 2/3, p. 839-849, 2004.

CIRIO, G. M.; LIMA, M. L. R. Z. C. Métodos de detecção do gênero Aspergillus em sementes de milho (Zea mays) em 270 dias de armazenamento. Visão Acadêmica, Curitiba, v. 4, n. 1, p. 19-23, 2003.

FARIAS, C .R. J.; LUCCA-FILHO, O. A.; PIEROBOM, C. R.; DELPONTE, E. M. Qualidade sanitária de sementes de aveia-preta (Avena strigosa Schreb.) produzidas no estado do Rio Grande do Sul, safra 1999/2000. Revista Brasileira de Sementes, Londrina, v. 24, n. 1, p. 1-4, 2002.

FERNANDES, C. D.; MARCHI, C. E.; JERBA, V. F.; BORGES, M. F. Patógenos associados às sementes de forrageiras tropicais e estratégias de controle. In: ZAMBOLIM, L.(Ed.). Sementes: qualidade fitossanitária. Viçosa, MG: UFV, p. 183-213. 2005.

GARCIA, J. W.; TENENTE, R. C. V. Reprodução de Aphelenchoides besseyi Christie (1942) obtido de sementes de Panicum maximum, em diferentes fungos associados a essas sementes. Nematologia Brasileira, Campinas, v. 24, n. 1, p. 91-93, 2000.

GOMES, D. P.; SILVA, G. C.; CAVALCANTE, M. R.; SILVA, A. C.; CÂNDIDO, C. S.; MACHADO, K. K. G.; RÊGO,A.S. Qualidade de sementes de Panicum maximum cv. Tanzânia e Brachiaria humidicola. In: ENCONTRO LATINO AMERICANO DE INICIAÇÃO CIENTÍFICA, 12., ENCONTRO LATINO AMERICANO DE PÓSGRADUAÇÃO - UNIVERSIDADE DO VALE DO PARAÍBA, 8., 2008, São José dos Campos. Anais... São José dos Campos: Univap, 2008. p. 1-3.

GUIMARÃES, L. R. A.; MARCHI, C. E.; FERNANDES, C. D.; JERBA, V. F.; BUENO, M. L.; TRENTIN, R. A.; FABRIS, L. R. Fungos associados às sementes comerciais de braquiária. In: JORNADA CIENTÍFICA DA EMBRAPA GADO DE CORTE, 2., 2006, Campo Grande. Anais... Campo Grande: Embrapa Gado de Corte, 2006. p. 1-2.

LASCA, C. C.; VECHIATO, M. H.; KOHARA, E. Y. Controle de fungos de sementes de Brachiaria spp.: eficiência de fungicidas e influência do período de armazenamento de sementes tratadas sobre a ação desses produtos. Arquivos do Instituto Biológico, São Paulo, v. 71, n. 4, p. 465-472, 2004.
MARCHI, C. E.; BORGES, M. F.; ARIAS, A. M. S.; FERNANDES, C. D.; JERBA, V. F.; BUENO, M. L. Patologia de sementes de forrageiras tropicais. Summa Phytopathologica, Botucatu, v. 32, p. 81, 2006 a. Suplemento.

MARCHI, C. E.; FERNANDES, C. D.; FABRIS, L. R.; JERBA, V. F.; SORGATTO, M. Incidência de Ustilago operta em sementes comerciais de braquiária. Arquivos do Instituto Biológico, São Paulo, v. 76, n. 1, p. 121-125, 2009.

MARCHI, C. E.; FERNANDES, C. D.; JERBA, V. F.; TRENTIN, R. A.; BUENO, M. L.; GUIMARÃES, L. R. A.; FABRIS, L. R. Sementes de forrageiras tropicais: patógenos associados e estratégias de controle. In: SIMPÓSIO BRASILEIRO DE PATOLOGIA DE SEMENTES, 9., 2006, Passo Fundo. Anais... Passo Fundo: Universidade de Passo Fundo, 2006b. 1 CDROM.

MENTEN, J. O. M. Prejuízos causados por patógenos associados às sementes. In: (Ed.). Patógenos em sementes: detecção, danos e controle químico. São Paulo: Ciba Agro, p. 115-136. 1995.

MICHALSKI, M. V.; CHARCHAR, M. J. d'A.; ANJOS, J. R. N.; FERNANDES, F. D.; SILVA, M. S.; SILVA, W. A. M. Transmissão de fungos de sementes para plântulas de Panicum maximum. In: ENCONTRO DE JOVENS TALENTOS DAEMBRAPACERRADOS, 3., Planaltina, 2007. Resumos... Planaltina: Embrapa Cerrados, 2007. p. 35. (Documentos Embrapa Cerrados, 176).

NEERGAARD, P. Seed pathology. London: The Macmillan, 1979. v. 1, 839 p.

SAS INSTITUTE. SAS/STAT software: changes and enhancements through release 9.1. Cary: Sas Institute, 2003. $5136 \mathrm{p}$.

TSUHAKO, A. T. Exportação de sementes de forrageiras tropicais. 2009. Disponível em: <http://www.matsuda. com.br/administracao/arquivo/Sementes\%20de\%20 Forrageiras\%20-\%20Seed\% 20News\%20abr\%202006. doc>. Acesso em: 30 abr. 2009.

URBEN, A. F. Fungos potencialmente patogênicos em germoplasma de forrageiras importado. In: SIMPÓSIO BRASILEIRO DE PATOLOGIA DE SEMENTES, 8., 2004, João Pessoa. Anais... João Pessoa: Universidade Federal da Paraíba, 2004, p. 127-131.

VECHIATO, M. H.; APARECIDO, C. C. Fungos em sementes de gramineas forrageiras: restriçãofitossanitária e métodos de detecção. São Paulo: Instituto Biológico de São Paulo, Comunicado Técnico, 89. 2008. Disponível em: $\quad<$ http://www.biologico.sp.gov.br/artigos_ok.php? id_artigo $=89>$. Acesso em: 01 abr. 2009. 